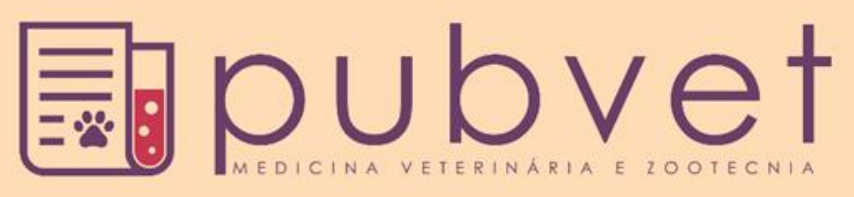

ISSN $1982-1263$

https://doi.org/10.31533/pubvet.v13n6a354.1-6

\title{
Comparação analgésica pós-operatória do tramadol ou morfina epidural em ovinos submetidos à cesariana
}

\author{
Luís Henrique Bedendo $^{1 *} \bullet$, Jean Carlos Gasparotto ${ }^{1}{ }^{\ominus}$, Charline Vanessa $\operatorname{Vaccarin}^{1}{ }^{\ominus}$, Hecson

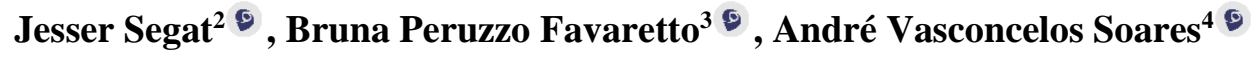 \\ ${ }^{1}$ Residente em Área Profissional da Saúde - Medicina Veterinária - Programa de Anestesia e Cirurgia Veterinária, Ênfase em Anestesiologia- \\ Universidade Federal de Santa Maria - UFSM, Santa Maria - RS Brasil \\ ${ }^{2}$ Dr. ciências biológicas com ênfase em bioquímica toxicológica - Programa Pós-graduação em Ciências Biológicas: Bioquímica toxicológica \\ UFSM; Santa Maria-RS- Brasil. \\ ${ }^{3}$ Mestranda Programa de Pós-Graduação medicina Veterinária - UFSM, Santa Maria - RS Brasil \\ ${ }^{4}$ Professor Adjunto de Anestesiologia Veterinária da UFSM-D Departamento de Pequenos Animais, Santa Maria - RS Brasil \\ *Autor para correspondência, E-mail: bedendo@upf.br
}

Resumo. Senciência, é a capacidade de sentir e engloba todos os animais vertebrados. No entanto, ruminantes, particularmente ovelhas, são seres relativamente estóicos, não apresentando sinais óbvios de angústia e dor. Objetivou-se avaliar a eficácia analgésica pós-operatória do uso de cloridrato de tramadol $2 \mathrm{mg} . \mathrm{kg}$-1 epidural (GT), ou sulfato de morfina $0,1 \mathrm{mg}_{\mathrm{kg}} \mathrm{kg}^{-1}$ epidural (GM), em ovelhas submetidas a cesariana. Após 60 minutos da administração da analgesia proposta via epidural, iniciava-se o procedimento cirúrgico, posicionados na mesa cirúrgica, em decúbito esternal, com olhos vedados. Após antissepsia realizava-se bloqueio paravertebral proximal, com agulha espinal longa, nos espaços T13L1, L1-L2 e L2-L3, depositando-se $2 \mathrm{ml}$ de cloridrato de lidocaína $2 \%$ sem vasoconstritor em cada ponto. A avaliação foi feita antes da deposição de opioide no espaço epidural (T0), e uma hora após a injeção (T1), duas (T2), três (T3), quatro (T4), seis (T6), oito (T8), dez (T10), doze (T12), dezesseis (T16), vinte (T20) e vinte e quatro horas (T24) pós administração epidural. O tempo cirúrgico foi de $112 \pm 30$ minutos. E utilizou-se adaptação da Escala Unidimensional da UNESP Botucatu para Avaliação da Dor Pós-operatória em Bovinos. Houve analgesia satisfatória até 8 horas do GT e 6 horas do GM. Não ocorreram alterações fisiológicas significativas. No entanto observou-se maior redução dos movimentos ruminais e respiratórios no GT. A adaptação da escala foi satisfatória na avaliação de dor.

Palavras-chave: analgesia, dor, peridural, escala, opioide, ovinos

\section{Postoperative analgesic comparison of tramadol or epidural morphine in sheep submitted to cesarean section}

Abstract. Sense, the ability to feel, encompasses all vertebrate animals. However,
ruminants, particularly sheep, are relatively stoic beings, showing no obvious signs of
anguish and pain, but they can feel it. The objective of this study was to evaluate the
postoperative analgesic efficiency of the use of $2 \mathrm{mg} . \mathrm{kg}-1$ epidural tramadol hydrochloride
(GT), or $0,1 \mathrm{mg} . \mathrm{kg}-1$ epidural morphine sulfate (GM), in ewes submitted to cesarean
section. After administration of the analgesia proposed via the epidural, it was expected 60
minutes to start the surgical procedure. The animals were evaluated before epidural
administration. The animals were placed on the surgical table, in sternal decubitus,
contained by limbs and eyelashes. After antisepsis, proximal paravertebral block with long
spinal needle was performed in the T13-L1, L1-L2 and L2-L3 spaces, and 2 ml of lidocaine
hydrochloride $2 \%$ without vasoconstrictor was deposited at each point. (T0), one hour after 
injection (T1), two (T2), three (T3), four (T4), six (T6), eight (T8), ten (T10), twelve (T12), sixteen T16), twenty (T20) and twenty-four hours (T24) post epidural administration. The surgical time was $112 \pm 30$ minutes. An adaptation of the UNESP Botucatu's OneDimensional Scale for Post-Operative Pain Evaluation in Bovine was used. There was satisfactory analgesia up to 8 GT hours and 6 hours GM. There were no significant physiological changes. However, there was a greater reduction of ruminal and respiratory movements in GT. Scale adaptation was satisfactory in the evaluation of pain.

Key words: analgesia, epidural, opioid, pain, scale, sheep

\section{Comparación analgésica postoperatoria del tramadol o morfina epidural en ovinos sometidos a cesárea}

Resumen. Senciencia, es la capacidad de sentir y engloba todos los animales vertebrados. Sin embargo, rumiantes, particularmente ovejas, son seres relativamente estoicos, no presentando signos obvios de angustia y dolor. Se objetivó evaluar la eficiencia analgésica posoperatoria del uso de hidrocloruro de tramadol $2 \mathrm{mg} \cdot \mathrm{kg}-1$ epidural (GT), o sulfato de morfina 0,1mg.kg-1 epidural (GM), en ovejas sometidas a cesárea. Después de la administración de la analgesia propuesta vía epidural, se esperaba 60 minutos para iniciar el procedimiento quirúrgico. Los animales eran colocados en la mesa quirúrgica, en decúbito esternal, contenidos por los miembros y con los ojos tapados. Posteriormente a la antisepsia se realizó el bloqueo paravertebral proximal, en los espacios T13-L1, L1-L2 y L2-L3, se depositó $2 \mathrm{ml}$ de hidrocloruro de lidocaína $2 \%$ sin vasoconstrictor en cada punto, con una aguja espinal larga. Se realizo una evaluación antes de la deposición de opioide em el espacio epidural (T0), una hora después de la inyección (T1), dos (T2), tres (T3), cuatro (T4), seis (T6), ocho (T8), diez (T10), doce (T12), dieciséis (T16), veinte (T20) y veinticuatro horas (T24) después de la administración epidural. El tiempo quirúrgico fue de $112 \pm 30$ minutos. Se utilizó adaptación de la Escala Unidimensional de la UNESP Botucatu para Evaluación del dolor Posoperatoria en Bovinos. Se observó una analgesia satisfactoria hasta 8 horas del GT y 6 horas del GM. No hubo cambios fisiológicos significativos. Sin embargo, se observó una mayor reducción de los movimientos ruminal y respiratorio en el GT. La adaptación de la escala fue satisfactoria en la evaluación del dolor.

Palabras clave: analgesia, dolor, peridural, escala, opioide, ovinos

\section{Introdução}

Com o avanço da ciência do bem-estar animal, tem-se aguçado o senso crítico quanto a necessidade de prevenção e tratamento da dor em animais. Desta forma, o bem-estar animal tem passado de um empecilho às práticas de produção, a um aliado importante para a viabilidade financeira do agronegócio, agregando valor ao produto (Luna, 2006).

Ovinos, como outros ruminantes, manifestam dor após estímulos nocivos decorrentes de procedimentos de manejo ou como resultado direto ou indireto de anormalidades fisiológicas. A administração epidural de opioides minimiza o trajeto e as barreiras fisiológicas para alcançar os receptores espinhais, permitindo o uso de doses menores comparando-se às vias parenterais, com redução dos efeitos adversos (Madureira, 2018).

O presente trabalho tem por objetivo avaliar a eficácia analgésica pós-operatória do cloridrato de tramadol $2 \mathrm{mg} \cdot \mathrm{kg}^{-}{ }^{1}$ ou do sulfato de morfina $0,1 \mathrm{mg} \cdot \mathrm{kg}_{-}{ }^{1}$, ambos diluídos para volume total de $5 \mathrm{ml}$ com água para injeção e administrados via epidural.

A ausência de escala de dor em ovinos dificulta a avaliação dos mesmos, levando a subestimação da dor, e seu correto tratamento. No presente projeto utilizou-se adaptação da escala unidimensional de dor aguda de bovinos UNESP - Botucatu. No entanto existem particularidades não aplicas a espécie ovina. 


\section{Material e métodos}

O presente trabalho desenvolvido no Hospital Veterinário Universitário da Universidade Federal de Santa Maria, no setor de ruminantes, tendo sido aprovado pelo CEUA-UFSM sob parecer $\mathrm{n}^{\circ}$ 1971250618. Avaliou-se a dor pós-operatória de cinco ovelhas, mestiças Texel, entre dois e quatro anos, com peso corporal de $54 \pm 8 \mathrm{~kg}$ submetidas a cesariana. Os animais foram avaliados 10, 5, 3, 2 e 1 dias antes do procedimento para aclimatação e redução do estresse do manejo causado pelos avaliadores. Um dia antes da data prevista para a cirurgia de cesariana, realizou-se a tricotomia do flanco esquerdo e da região coxígea.

Os animais foram alocados aleatoriamente em dois grupos, sendo eles: Grupo Morfina (GM, n=3) que recebeu $0,1 \mathrm{mg} . \mathrm{kg}^{-1}$ morfina e o Grupo Tramadol (GT, $\mathrm{n}=2$ ) que recebeu $2 \mathrm{mg} \cdot \mathrm{kg}^{-1}$ de Cloridrato de Tramadol, ambos os grupos perfizeram um volume de administração de $5 \mathrm{ml}$ em diluição com água destilada e tiveram a administração realizada no espaço epidural sacro coxígeo. $O$ correto posicionamento da agulha de Tuohy era confirmado através da perda de resistência a administração de ar com seringa de vidro, respeitando as boas práticas de antissepsia. Após injeção epidural, os animais receberam penicilina associada ao piroxicam por via intramuscular ajustados para 10000 UI.kg-1 de penicilina procaína. Após uma hora os animais eram posicionados na mesa cirúrgica, em decúbito esternal, com olhos vendados, uma vez que estavam conscientes. Após antissepsia realizava-se o bloqueio paravertebral proximal, com agulha espinhal longa, nos espaços T13-L1, L1-L2 e L2-L3, depositando-se $2 \mathrm{ml}$ de cloridrato de lidocaína $2 \%$ sem vasoconstritor. Posteriormente era realizado pinçamento da pele para testar bloqueio.

O tempo cirúrgico foi de $112 \pm 30$ minutos. Os animais foram avaliados quanto: frequência cardíaca (FC), frequência respiratória $(f)$, presença de midríase, movimentos ruminais, e comportamento, antes da deposição de opioide no espaço epidural (T0), uma hora após a injeção (T1), duas (T2), três (T3), quatro (T4), seis (T6), oito (T8), dez (T10), doze (T12), dezesseis (T16), vinte (T20) e vinte e quatro horas (T24) pós administração epidural. Utilizou-se uma adaptação da Escala Unidimensional da UNESP Botucatu para Avaliação da Dor Pós-operatória em Bovinos (Oliveira, 2014). Os animais que obtiveram pontuação maior ou igual a 5 na escala receberiam resgate analgésico com $2 \mathrm{mg} . \mathrm{kg}-1 \mathrm{de}$ cloridrato de tramadol IM e dipirona $25 \mathrm{mg} . \mathrm{kg}-1 \mathrm{IM}$ e seriam avaliados novamente em 20 minutos e até que atingissem valores condizentes com ausência de dor, na avaliação analgésica.

Apesar de ovinos serem ruminantes assim como bovinos, existem peculiaridades entre as espécies. Para tal, adaptou-se a escala de avaliação de dor aguda em bovinos (figura 1) a fim de obter maior precisão na avaliação.

\begin{tabular}{|c|c|c|c|}
\hline \multicolumn{2}{|c|}{ Escala adaptada de Botucatu } & 0 & 1 \\
\hline \multicolumn{2}{|l|}{ hora } & & \\
\hline Locomoção & $\begin{array}{l}\text { (0) move-se livremente, sem alteraça de locomoção: } \\
\text { 1. J Move-se com restricão, e ao andar a linha dorsal } \\
\text { pode estar normal ou arqueada, e os passos podem } \\
\text { estar mais curtos } \\
\text { Reluta em se levantar ou quando se levanta, o faz } \\
\text { com dificuldade, ou não se locomove }\end{array}$ & & \\
\hline $\begin{array}{l}\text { II } \\
\text { Interação } \\
\text { com o } \\
\text { ambiente }\end{array}$ & $\begin{array}{l}\text { (0) ativo, atento aos estímulos ambientais táteis e/ou visuais } \\
\text { e/ou sonoros } \\
\text { (1) Apático, quando estimulado, interage pouco } \\
\text { (2 apático, não reage aos estímulos ambientais táteis, visuais } \\
\text { e/ou sonoros }\end{array}$ & & \\
\hline $\begin{array}{l}\text { III } \\
\text { Atividade }\end{array}$ & $\begin{array}{l}\text { O- Movimenta-se normalmente } \\
\text { Inquieto, movimenta-se acima do normal, ou deita e } \\
\text { levanta com frequência } \\
\text { Movimenta-se com menor frequência ou somente } \\
\text { quando estimulado }\end{array}$ & & \\
\hline $\begin{array}{l}\text { IV } \\
\text { Ingestão de } \\
\text { alimentos }\end{array}$ & $\begin{array}{l}\text { O- Normorexia e/ou ruminação presente } \\
\text { 1. Hiporexia } \\
\text { 2. Anorexia / Ranger dos dentes }\end{array}$ & & \\
\hline $\begin{array}{l}\text { M- } \\
\text { Miscelânea } \\
\text { comportam } \\
\text { entos }\end{array}$ & 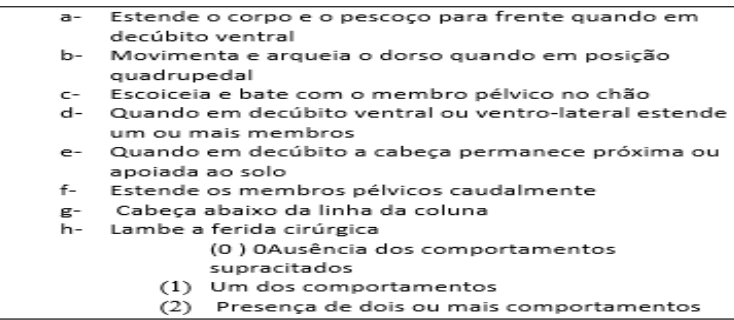 & & \\
\hline
\end{tabular}

Figura 1. Escala adaptada para ovinos da "Escala Unidimensional da UNESP-Botucatu para avaliação de dor aguda pós-operatória em bovinos. 
Para as análises estatísticas foi realizado teste ANOVA de duas vias com medida repetida, seguida de teste post-hoc de Fischer (Software package Statistic 8.0 para Windows). Valores de $\mathrm{P} \leq 0,05$ foram considerados estatisticamente significantes para as comparações realizadas.

\section{Resultados e discussão}

Dos cinco animais avaliados, dois animais não apresentaram dor até as 24 horas após a cirurgia, dois até as 8 horas, um de cada grupo respectivamente, e um até as 6 horas (GM) pós administração de morfina epidural. No entanto houve variação entre o tempo cirúrgico em ambos os grupos, o que pode ter interferido nos resultados. Segundo Baniadam et al. (2010) a analgesia induzida pela administração de tramadol coxigea em vacas foi dependente da dose (por exemplo, a duração da analgesia completa no períneo foi de 18 minutos quando as vacas receberam a dose de $1 \mathrm{mg} / \mathrm{kg}, 60$ minutos quando receberam a dose de $2 \mathrm{mg} / \mathrm{kg}$, e 92 minutos quando receberam a dose de $3 \mathrm{mg} / \mathrm{kg}$. Sedação leve a moderada e ataxia foram observadas quando as vacas receberam 2 ou $3 \mathrm{mg} . \mathrm{kg}$ de tramadol.

O GT apresentou escores de dor inferiores aos do GM até às quatro horas pós-injeção, três horas após o início da cirurgia, no entanto o GM manteve-se mais estável até às 8 horas após a injeção epidural de opioide (Figura 2). Para Robinson \& Natalini (2002) em equinos, submetidos a procedimentos cirurgicos na posiçao quadrupedal, a falta de comprometimento motor e a analgesia profunda produzida pela morfina e tramadol sugerem que essas drogas podem ser combinadas com anestésicos locais, como a lidocaína, para produzir um efeito duradouro na anestesia cirúrgica e analgesia pós-operatória prolongada sem ataxia ou em decúbito, permitindo pronta recuperação do paciente ao término do procedimento, conforme o ocorrido nas ovelhas, permitindo a rápida aceitação das proles. Um animal do GM recebeu resgate após seis horas da injeção epidural, por apresentar escore de dor superior a cinco. Dois animais necessitaram de resgate analgésico com 8 horas após administração epidural, um de cada grupo. 30 minutos após o resgate, todos os animais apresentaram escore de dor inferior a 5, mostrandose satisfatório o protocolo de resgate. Todos os animais foram diariamente avaliados até a alta clínica quanto a dor, e as medicações pertinentes foram administradas conforme a necessidade.

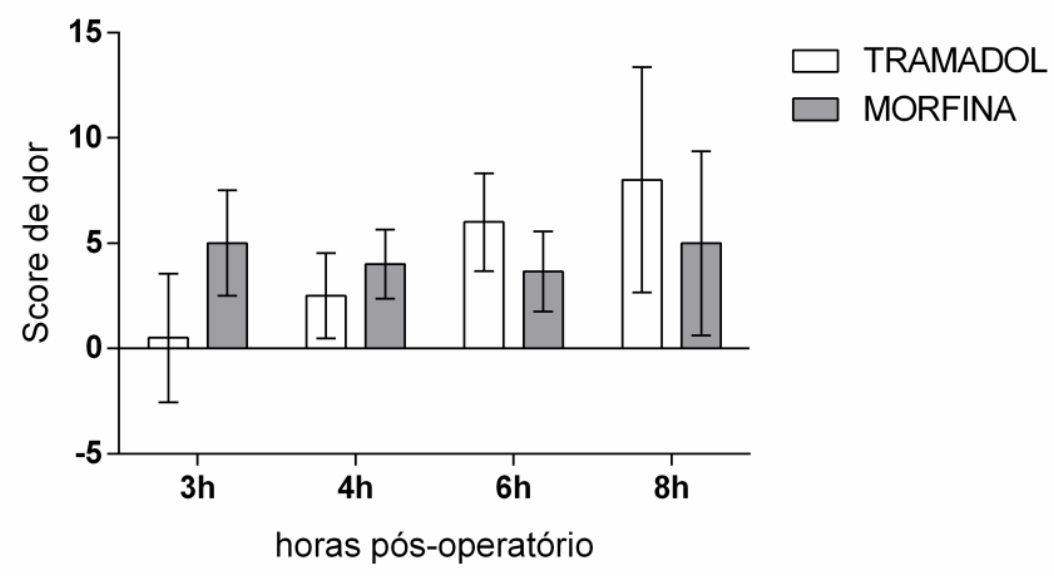

Figura 2. Avaliação pós-operatória do escore de dor em ovinos submetidos à cesariana, após aplicação de protocolos anestésicos contendo tramadol ou morfina. Os dados foram apresentados como média \pm E.P.M $(\mathrm{P} \leq 0,05)$.

Segundo Baniadam et al. (2010) a administração epidural de tramadol em vacas reduziu a motilidade ruminal, porém sem repercussões clínicas. No presente estudo também se observou redução na motilidade ruminal (cerca de 50\%) (Figura 3), porém sem repercussão clínica relevante aos animais. O GT apresentou maior redução da motilidade. Apesar de Madureira (2018) ter observado aumento da motilidade ruminal após injeção epidural de morfina $0,1 \mathrm{mg} / \mathrm{kg}$, o fato pode estar relacionado a melhora do quadro clínico do animal do estudo. 
A

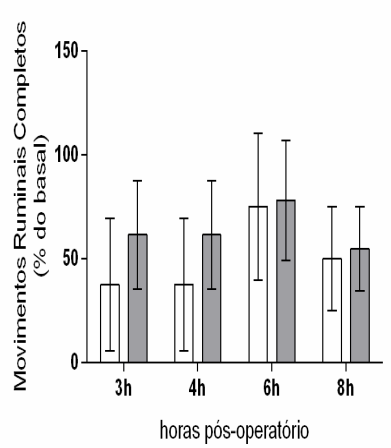

$B$

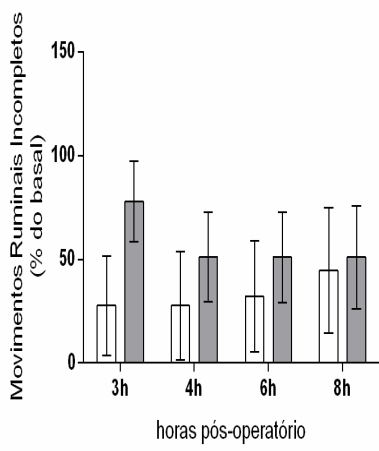

C

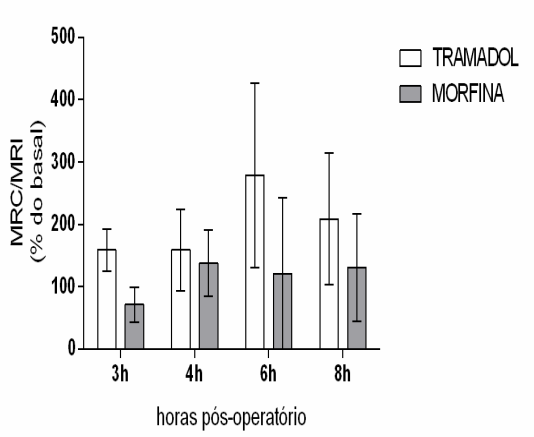

Figura 3. Avaliação pós-operatória dos movimentos ruminais completos (A), incompletos (B) e razão entre movimentação ruminal completa e incompleta $(\mathrm{C})$, em ovinos submetidos à cesariana, após aplicação de protocolos anestésicos contendo tramadol ou morfina.

Observou-se grande variação nas frequências cardíaca (FC) e respiratória $(f)$, entre animais no T0 (basal), acreditando-se se tratar de variação individual, assim como afirma Madureira (2018), quando cita que esta espécie apresenta grande variação da $f$ em função da regulação térmica ou do estresse. Devido ao pequeno $\mathrm{n}^{\circ}$ amostral, trabalhou-se apenas com a porcentagem da diferença de valores de cada animal em relação ao T0. Por exemplo, o animal um tinha 74 bpm, no T0, e 96bpm no T2, marcando um aumento de 30\%. Já o animal cinco, tinha 168 bpm no T0, e 204 bpm no T4, valores bem superiores ao do primeiro animal, mas aplicando-se a regra, houve um aumento de apenas $21 \%$ na frequência. Assim evitou-se que a variação individual interferisse na avaliação estatística.

Houve aumento nas frequências cardíacas (bpm) de ambos os grupos. Já a frequência respiratória (mpm) teve aumento com o grupo morfina, e redução com o GT comparados aos valores basais de cada animal (figura 4). Discordando de Baniadam et al. (2010) e Madureira (2018) que não encontraram diferenças nas frequências cardíacas e respiratórias.
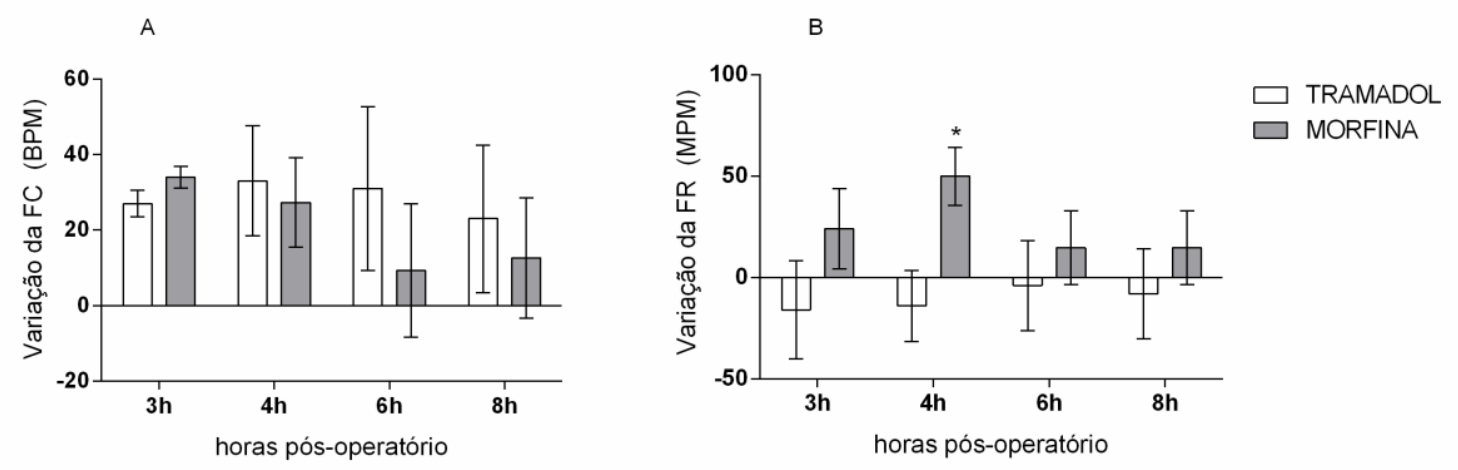

Figura 4. Variação da Frequência cardíaca (A) e variação da frequência respiratória (B) no período pós-operatório, após aplicação de protocolos anestésicos contendo tramadol ou morfina. Os dados foram apresentados como média \pm E.P.M $(\mathrm{p} \leq 0,05)$.

Durante as últimas duas a três décadas houve uma evolução gradual quanto ao desenvolvimento de novas técnicas veterinárias para o controle da dor dos animais. Assim sendo, o aumento do uso de analgésicos tem sido acompanhado de melhorias na capacidade de avaliar a dor nestes pacientes. Os comportamentos expressos por muitas espécies, no entanto, serão muito diferentes daquelas observadas em seres humanos e, em algumas circunstâncias, pode ser mascarado completamente pela resposta dos animais ao serem observados. Esta carência de respostas facilmente identificáveis à dor pode levar à suposição de que a dor significativa não pode ser presente. Este obstáculo para o controle eficaz da dor pode só ser superado pelo desenvolvimento de escalas de pontuação (Flecknell, 2008). 
Não é suficiente apenas supor que os animais sintam dor, sem critérios e escalas para direcionar a avaliação. A administração de fármacos analgésicos baseados na suposição, além de proporcionar falhas na mensuração, administrações desnecessárias ou insuficientes ainda pode submeter o paciente a riscos (Flecknell, 2008).

Devido à falta de escala específica para ovinos até o momento da confecção do trabalho, usou-se a extrapolação da escala desenvolvida para bovinos pela universidade de Botucatu.

\section{Conclusão}

O tramadol foi eficaz até 7 horas de pós-cirúrgico, a morfina foi eficaz até as 5 horas pós cirúrgicos (oito e seis horas da aplicação epidural respectivamente). Apesar da literatura mencionar analgesia da morfina de até 24 horas, houve animais que necessitaram de resgate em período muito inferior, ressaltando a necessidade de avaliação constante da dor.

A escala utilizada foi capaz de quantificar e orientar a percepção de dor pós-operatória em ovinos. No entanto, esforços não devem ser medidos e escalas específicas devem ser desenvolvidas e validadas para observação espécie-específicas como a em estudo.

\section{Referências bibliográficas}

Baniadam, A., Afshar, F. S. \& Ahmadian, F. (2010). Analgesic effects of tramadol hydrochloride administered via caudal epidural injection in healthy adult cattle. American Journal of Veterinary Research, 71(7):720-725. doi: https://doi.org/10.2460/ajvr.71.7.720.

Flecknell, P. A. (2008). Analgesia from a veterinary perspective. British Journal of Anaesthesia, 101(1):121-124. doi: https://doi.org/10.1093/bja/aen087.

Luna, S. P. L. (2006). Dor e sofrimento animal. In E. A. B. Rivera, M. H. Amaral \& V. P. Nascimento (Eds.), Ética e bioética (pp. 131-158). Goiânia, Goiás, Brasil.

Madureira, K. M. (2018). Morfina epidural contínua no controle da dor pós-operatória em ovino. Acta Scientiae Veterinariae, 46(4):1-6.

Oliveira, F. A. de. (2014). Desenvolvimento, validação e confiabilidade de uma escala de dor aguda pós-operatória em bovinos. Faculdade de Medicina de Botucatu, UNESP. Retrieved from https://repositorio.unesp.br/bitstream/handle/11449/114042/000798948.pdf?sequence=1\&isAllowed=y

Robinson, E. P. \& Natalini, C. C. (2002). Epidural anesthesia and analgesia in horses History of epidural analgesia and anesthesia in horses, 18, 61-82. Veterinary Clinics of North America: Equine Practice, 18(1):61-82.

Recebido: 24 de março, 2019

Aprovado: 15 de maio, 2019.

Publicado: 30 de junho, 2019.

Licenciamento: Este artigo é publicado na modalidade Acesso Aberto sob a licença Creative Commons Atribuição 4.0 (CCBY 4.0), a qual permite uso irrestrito, distribuição, reprodução em qualquer meio, desde que o autor e a fonte sejam devidamente creditados. 